Cite this: Soft Matter, 2014, 10, 5729

\title{
Two glass transitions of polyurea networks: effect of the segmental molecular weight
}

\author{
Marius Reinecker, ${ }^{\text {*a }}$ Viktor Soprunyuk, ${ }^{a}$ Martin Fally, ${ }^{a}$ Antoni Sánchez-Ferrer ${ }^{\star b}$ \\ and Wilfried Schranz ${ }^{* a}$
}

Polymer-nanoparticle composites (PNCs) play an increasing role in technology. Inorganic or organic nanoparticles are usually incorporated into a polymer matrix to improve material properties. Polyurea is a spontaneously occurring PNC, exhibiting a phase segregated structure with hard nanodomains embedded in a soft (elastically compliant) matrix. This system shows two glass transitions at $T_{\mathrm{g} 1}$ and $T_{\mathrm{g} 2}$. It has been argued that they are related to the freezing of motion of molecular segments in the soft matrix (usual polymer $\alpha$-glass transition at $T_{\mathrm{g} 1}$ ) and to regions of restricted mobility near the hard nanodomains ( $\alpha^{\prime}$-process) at $T_{\mathrm{g} 2}$, respectively. We present detailed dynamic mechanical analysis (DMA) measurements for polyurea networks with different segmental lengths $l_{c}(2.5,12.1,24.5 \mathrm{~nm})$ of the polymer chains, i.e. different volume fractions $\phi_{x}(0.39,0.12,0.07)$ of the hard domains. The two glass transitions show up in two distinct peaks in $\tan \delta$ at $T_{\alpha}$ and $T_{\alpha^{\prime}}$. Analysing the data using a HavriliakNegami term for the $\alpha$ - and $\alpha^{\prime}$-relaxation, as well as Vogel-Fulcher dependencies for the corresponding relaxation times, it is found that the $\alpha$-glass transition at $T_{\mathrm{g} 1}$ increases strongly (up to $\Delta T=70 \mathrm{~K}$ ) with increasing $\phi_{x}$, whereas the $\alpha^{\prime}$-transition at $T_{\mathrm{g} 2}$ remains unchanged. At $\phi_{\mathrm{x}}^{\mathrm{c}} \approx 0.19$ the two curves intersect, i.e. $T_{\mathrm{g} 1}=T_{\mathrm{g} 2}$. This value of $\phi_{\mathrm{x}}^{\mathrm{c}}$ is very close to the percolation threshold of randomly oriented overlapping ellipsoids of revolution with an aspect ratio of about $1: 4-1: 5$. We therefore conclude that around $19 \%$ of the hard nanodomains polyurea changes from a system of hard nanoparticles embedded in a soft matrix $\left(\phi_{\mathrm{x}} \leq \phi_{\mathrm{x}}^{\mathrm{c}}\right)$ to a system of soft domains confined in a network of percolated hard domains at $\phi_{\mathrm{x}} \geq \phi_{\mathrm{x}}^{\mathrm{c}}$.

Received 6th May 2014 Accepted 2nd June 2014

DOI: $10.1039 / c 4 s m 00979 g$

www.rsc.org/softmatter

\section{Introduction}

The glass transition remains one of the great mysteries in material science despite of decades of intense research. According to the work of Adam and Gibbs ${ }^{1}$ the generally observed drastic slowing down of the dynamics around $T_{\mathrm{g}}$ originates from so-called "cooperatively rearranging regions (CRRs)", whose size $\xi$ increases when $T$ approaches $T_{\mathrm{g}}$. The corresponding relaxation time $\tau_{\alpha}(\xi)$ is given by ${ }^{2}$

$$
\tau_{\alpha}(\xi)=\tau_{0} \exp \left[\xi(T)^{D} \frac{\Delta}{\kappa_{\mathrm{B}} T}\right]
$$

where $\Delta$ is a typical energy scale that depends on the details of the glass former and is traditionally assumed to be constant. $\xi(T)$ contains the main $T$ dependence and $D$ is the spatial dimension of correlated regions. The glass transition temperature $T_{\mathrm{g}}$ (so-called "laboratory glass transition temperature") is usually defined for $\tau_{\alpha} \approx 100 \mathrm{~s}$ or shear viscosities in the range of about $10^{13}$ poise. $^{3}$ The main question, whether there is a true

${ }^{a}$ Faculty of Physics, University of Vienna, Boltzmanngasse 5, 1090 Vienna, Austria. E-mail: marius.reinecker@univie.ac.at; wilfried.schranz@univie.ac.at

${ }^{b}$ Department of Health Sciences and Technology, ETH Zurich, Institute of Food, Nutrition and Health, Food and Soft Materials Science Group, Schmelzbergstrasse 9, LFO, E29, 8092 Zurich, Switzerland.E-mail: antoni.sanchez@hest.ethz.ch thermodynamic phase below $T_{\mathrm{g}}$, or if it is just a kinetic slowing down at $T=0 \mathrm{~K}$, is intimately related to the question of a diverging correlation length $\xi$ at a finite temperature $T_{0}$ below $T_{\mathrm{g}}$. Substantial progress has been made in recent years to determine such length scales. Quite different experimental techniques, such as heat capacity spectroscopy, ${ }^{4}$ multidimensional $\mathrm{NMR}^{5,6}$ dielectric spectroscopy ${ }^{7}$ as well as computer simulations ${ }^{8}$ have been used to monitor a possible growing length scale accompanying the glass transition in various glass forming materials including molecular liquids and polymers.

It is now generally accepted that the nature of cooperativity is dynamic and the obtained dynamically correlated regions $\xi$ are increasing when approaching $T_{\mathrm{g}}$. However, it is very difficult to nail down quantitative values for $\xi$ exactly. To a great extent this is due to the unknown shape of correlated regions, which can change from compact to string like structures with changing temperature..$^{\mathbf{8} 9}$

An appealing method to probe correlation lengths in glass forming materials is to restrict their spatial extent $d$. The basic idea is the following: if a growing correlation length $\xi(T)$ for $T \rightarrow$ $T_{\mathrm{g}}$ would be involved in the glass transition, the system should start to "feel" the spatial confinement as soon as $\xi\left(T_{\mathrm{g}}\right) \approx d$, and the glass transitions should be finally suppressed for $d<\xi\left(T_{\mathrm{g}}\right)$. Indeed, numerous measurements of confined glass forming 
materials, i.e. of thin polymer films, ${ }^{\mathbf{1 0}, \mathbf{1 1}}$ molecular liquids in nanopores, ${ }^{12-14}$ as well as computer simulations ${ }^{\mathbf{1 5 , 1 6}}$ support this idea. In most cases confinement leads to a remarkable downshift of $T_{\mathrm{g}}$, due to so-called "confinement induced acceleration of the dynamics". There are however also cases where a gradual increase of the glass transition temperature with decreasing pore size was reported. ${ }^{17}$ It turns out that attractive interactions between the confined glass former and the pore surface can slow down the motion of molecules near the surface, which due to an increase in the correlation length can slow down larger regions, leading to an effective upshift of $T_{\mathrm{g}}$. Interestingly it was found, ${ }^{\mathbf{1 8 , 1 9}}$ that a second glass transition at $T_{\mathrm{g} 2}>T_{\mathrm{g}}$ can occur if the interfacial region of slowed down motion is large enough, i.e. of the order of the correlation volume $V_{\text {corr }}\left(T_{\mathrm{g}}\right)$ of dynamically correlated regions. Effects of confinement on material behaviour including freezing and melting have been discussed in excellent review articles. ${ }^{20,21}$

Exciting interfacial phenomena have been also studied by incorporation of nanoparticles into a polymer host. ${ }^{22}$ Depending on the type of interaction (attractive or repulsive) between the polymer chains and the nanoparticles, the relaxation dynamics of the polymer chains close to the nanoparticles may increase (for repulsive interactions) or decrease (for attractive interactions), leading to a down- or upshift of $T_{\mathrm{g}}$, respectively. ${ }^{23,24}$ In the case of permanent attachment of polymer chains to nanoparticles one can even obtain two glass transitions: ${ }^{25}$ one at $T_{\mathrm{g} 1}$ associated with polymer chains far from the nanoparticles and a second $T_{\mathrm{g} 2}>T_{\mathrm{g} 1}$ associated with chain segments in the vicinity of the nanoparticles. Indeed such polymer nanocomposites (PNCs) are in many respects similar to confined pure polymers or liquid glass formers in nanopores. ${ }^{26}$

Polyurea is a unique material, ${ }^{27}$ since it offers the possibility to study the influence of nanoparticles on the glass dynamics without the need to add nanoparticles due to its microphase segregated structure into hard and soft nanodomains, whose volume ratios can be easily varied by changing the molecular weight of the polyetheramines. The type of interaction (attractive $v s$. repulsive) between the polymer chains near the hard nanodomains is clearly attractive, since the polymer chains are chemically attached to the hard nanodomains. Because of the complex microstructure polyureas display a very broad range of mechanical responses under static and dynamic loading conditions, which favour their use as abrasion/corrosion protection and blast/ballistic-impact mitigation materials.

In the present work we use samples of polyurea networks with three different segmental lengths $l_{\mathrm{c}}$ (Table 1) of the

Table 1 Segmental molecular weight $\left(\bar{M}_{c}\right)$ of polyetheramines, segmental length $\left(l_{c}\right)$, volume fractions $\left(\phi_{x}\right)$ of the crosslinker (hard domains) and average distance $t$ between hard domains for all three polyurea elastomers

\begin{tabular}{lcccc}
\hline Sample & $\bar{M}_{\mathrm{c}}\left(\mathrm{g} \mathrm{mol}^{-1}\right)$ & $l_{\mathrm{c}}(\mathrm{nm})$ & $\phi_{\mathrm{x}}$ & $t(\mathrm{~nm})$ \\
\hline JD-400 & 428 & 2.5 & 0.39 & 4.8 \\
JD-2000 & 1990 & 12.1 & 0.12 & 5.4 \\
JD-4000 & 4024 & 24.5 & 0.07 & 7.5
\end{tabular}

polymer backbone (i.e. polyetheramine in our case) resulting in three different volume fractions $\phi_{\mathrm{x}}$ of the hard nanodomains and perform dynamic mechanical analysis measurements (DMA) as a function of temperature and frequency. Two successive glass transition temperatures at $T_{\mathrm{g} 1}$ and $T_{\mathrm{g} 2}$ are found and are attributed to cooperative motions of molecular segments in the soft matrix $\left(T_{\mathrm{g} 1}\right)$ and to regions in the vicinity of the hard domains $\left(T_{\mathrm{g} 2}\right)$, respectively.

Fitting a Havriliak-Negami function with the Vogel-Fulcher relaxation time behaviour to the temperature and frequency dependence of the experimental tan $\delta$ curves, we determine the glass transition temperatures $T_{\mathrm{g} 1}\left(\phi_{\mathrm{x}}\right)$ and $T_{\mathrm{g} 2}\left(\phi_{\mathrm{x}}\right)$ for various concentrations $\phi_{\mathrm{x}}$ of hard nanodomains.

From the experimental data of Young's modulus vs. temperature and frequency $E^{\prime}(\omega, T)$ we determine the temperature dependence of the linear size $\xi$ and volume $V_{\text {corr }}$ of dynamically correlated regions using the multipoint susceptibility approach of Berthier, et al. ${ }^{7}$ We show that these obtained values are consistent with the hypothesis ${ }^{25}$ that the regions of restricted mobility near the hard domains have to exceed a critical size of the order of $V_{\text {corr }}\left(T_{\mathrm{g}}\right)$ to exhibit their own glass transition at $T_{\mathrm{g} 2}$. Moreover, the obtained values of $\xi(T)$ turn out to be large enough to explain the observed increase of $T_{\mathrm{g} 1}$ with increasing volume fraction of hard nanodomains.

\section{Experimental}

\section{A Samples}

For the present study, the three linear hydrophobic diaminoterminated polyetheramines Jeffamine D-400 $\left(M_{\mathrm{n}}=460 \mathrm{~g}\right.$ $\left.\mathrm{mol}^{-1}, \rho=972 \mathrm{~kg} \mathrm{~m}^{-3}\right), \mathrm{D}-2000\left(M_{\mathrm{n}}=2060 \mathrm{~g} \mathrm{~mol}^{-1}, \rho=991 \mathrm{~kg}\right.$ $\left.\mathrm{m}^{-3}\right)$ and D-4000 $\left(M_{\mathrm{n}}=4000 \mathrm{~g} \mathrm{~mol}^{-1}, \rho=994 \mathrm{~kg} \mathrm{~m}^{-3}\right)$ from Huntsman International LLC, and the triisocyanate crosslinker Basonat HI-100 $\left(M_{\mathrm{w}}=504 \mathrm{~g} \mathrm{~mol}^{-1}, \rho=1174 \mathrm{~kg} \mathrm{~m}^{-3}\right)$ from BASF $\mathrm{SE}$ were used as received. Polyurea (PU) networks are formed by the rapid chemical reaction between a triisocyanate and a diamine to build urea moieties, as shown in Fig. 1. Details of the synthesis of these PU elastomers are well described in the literature. ${ }^{27}$ Table 1 compares the main structural characteristics for the three used samples JD-400, JD-2000 and JD-4000.

The structure of polyurea chains is shown in Fig. 1 (top). U-R$\mathrm{U}$ represents the hard segment (HS) of the polyurea molecule and $\mathrm{R}^{\prime}$ the soft segment (SS), and thus we can represent the molecules as shown in the bottom of Fig. 1.

Due to strong hydrogen bonding between urea linkages of neighboring chains or neighboring segments in the same chain, hard segments (HSs) are usually microphase segregated into socalled "hard domains".

Atomic force microcopy ${ }^{28}$ investigations revealed islands of hard domains embedded in the soft matrix where the average distance between hard domains is of the order of several nm (Table 1).

For small volume fractions of hard nanodomains the topology of phase segregated polyurea is "inverse" of the case of molecular liquids confined in meso- or nano-porous silica frameworks like Vycor or Gelsil. In these systems we recently studied the glass transitions of various glass forming liquids 

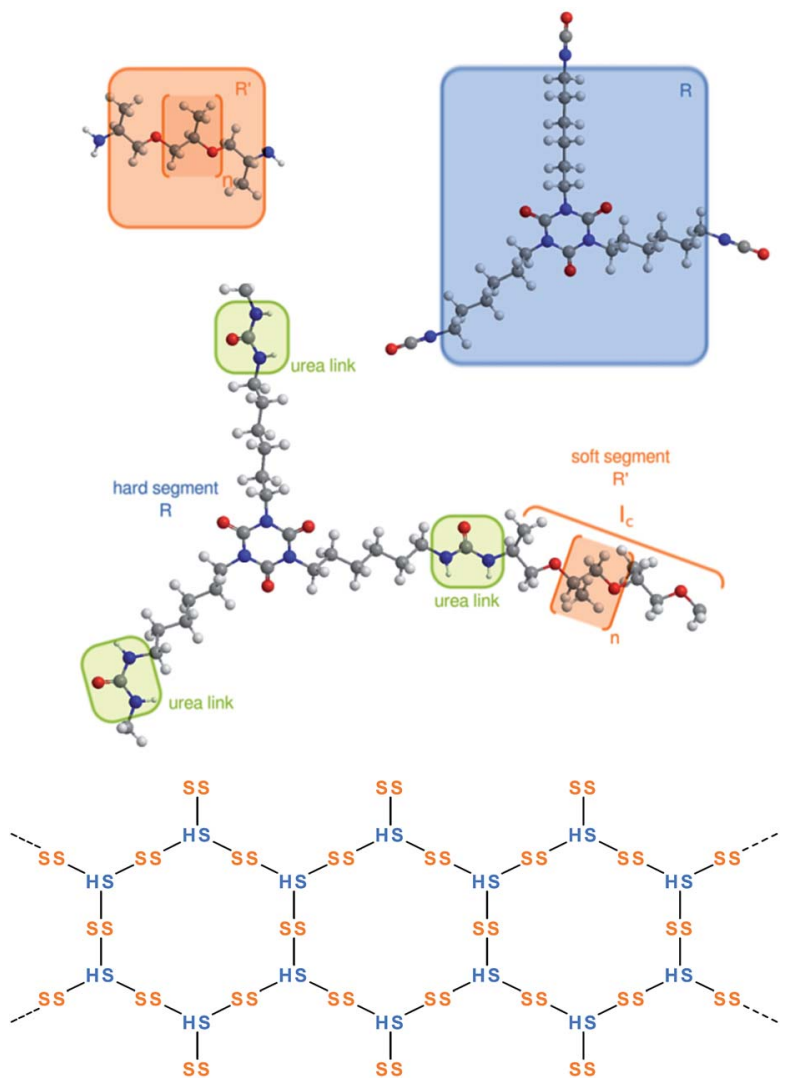

Fig. 1 Representation of a typical polyurea chain (top). R represents triisocyanate crosslinker Basonat and $\mathrm{R}^{\prime}$ denotes the diamino-terminated polyetheramine Jeffamine. The sketch at the bottom shows polyurea molecules built of hard and soft segments.

with varying pore diameters. ${ }^{29,30}$ E.g. for salol confined in nanoporous silica we detected ${ }^{29}$ two glass transitions for pore sizes larger than $5 \mathrm{~nm}$. We could associate the glass transition at $T_{\mathrm{g}_{2}}>T_{\mathrm{g} 1}$ with regions of restricted mobility near the surface of the pores. The glass transition at $T_{\mathrm{g} 1}$ was attributed to regions well inside the pores. Its dynamics was however also slowed down by surface interactions. This could be proved by silanization of the pore surface: removing the surface interactions resulted in a stronger decrease (as compared to untreated pores) of the bulk glass transition $T_{\mathrm{g} 1}$ with decreasing pore size and the second glass transition at $T_{\mathrm{g} 2}$ disappeared. ${ }^{30}$

In polyurea one obtains the inverse geometrical case for small enough volume fractions $\phi_{\mathrm{x}}$ of hard domains, as the elastically hard domains are suspended in the elastically compliant (soft) matrix. However, with increasing $\phi_{\mathrm{x}}$ we can expect a crossover to a system that is very similar to the case of glass forming liquids in nanopores.

\section{B Experimental methods}

Dynamic mechanical analysis (DMA) is used to measure the temperature dependencies of the low frequency elastic moduli and damping of polyurea at frequencies between 0.1 and 100 $\mathrm{Hz}$. A static force $F_{\text {stat }}$ is sinusoidally modulated by a dynamic force $F_{\mathrm{dyn}} \sin (\omega t)$ at a chosen amplitude and frequency. The

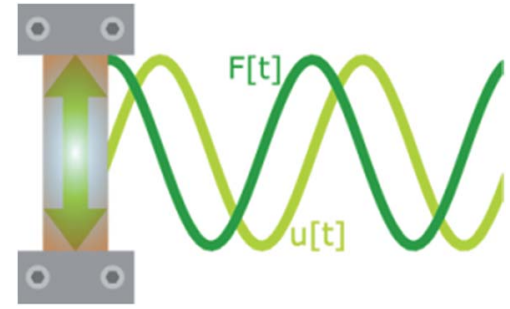

Fig. 2 Sketch of DMA measurement in tensile stress geometry.

elastic and anelastic response of the sample leads to a change in length $u$ and phase shift $\delta$ between force and response amplitude with resolutions for the length and phase shift of $\Delta u \approx 10$ $\mathrm{nm}$ and $\Delta \delta \approx 0.1^{\circ}$, respectively. The knowledge of the sample length $u$ and the phase shift $\delta$ allows us to determine the real and imaginary parts of a certain component of the complex elastic compliance tensor $S_{i i}^{*}$

$$
S_{i i}^{\prime}=S_{i i} \cos \delta \text { and } S_{i i}^{\prime \prime}=S_{i i} \sin \delta
$$

The corresponding storage $\left(E^{\prime}\right)$ and loss modulus $\left(E^{\prime \prime}\right)$ are defined as $E^{\prime}:=1 / S_{i i}^{\prime}$ and $E^{\prime \prime}:=1 / S_{i i}^{\prime \prime}$, respectively and $\tan \delta=E^{\prime \prime} / E^{\prime}$.

DMA experiments are performed on a Perkin Elmer Diamond DMA. The complex elastic compliance can be measured in a frequency range between $0.1 \mathrm{~Hz}$ and $100 \mathrm{~Hz}$, and for temperatures between $80 \mathrm{~K}$ and $850 \mathrm{~K}$. The minimal (maximal) force that can be applied is $10^{-3} \mathrm{~N}(10 \mathrm{~N})$ with a resolution of $10^{-5} \mathrm{~N}$. It is transmitted via a steel-rod. The present experiments are performed in tensile stress geometry (Fig. 2). For more details concerning the DMA method see e.g. ref. 31. The typical sample size was $9 \times 2 \times 0.2 \mathrm{~mm}^{3}$. The samples were cooled down to about $90 \mathrm{~K}$ and heated again with a heating rate between 0.5 and $2 \mathrm{~K} \mathrm{~min}^{-1}$. Up to 5 heating/cooling cycles were performed for each measurement frequency and each sample.

\section{Experimental results}

Fig. 3 shows the storage modulus $E^{\prime}$ and $\tan \delta$ of JD-2000 as a function of temperature at various frequencies.

In Fig. 4 and 5 the same quantities are plotted for JD-4000 and JD-400, respectively for $f=1 \mathrm{~Hz}$.

Inspecting Fig. 3-5 one recognizes quite similar behaviour for JD-2000 and JD-4000 samples. A typical relaxation behaviour of $E^{\prime}(\omega, T)$ together with the corresponding peak(s) in $\tan \delta$ whose maximum shifts to higher temperatures with increasing frequency. In JD-2000 and JD-4000 a second peak $\left(\alpha^{\prime}\right)$ is observed in $\tan \delta$ (Fig. 3 and 4). It can be seen in $E^{\prime \prime}$ data only if the scale is increased. The underlying process of this $\alpha^{\prime}$-relaxation will be discussed in detail below. Note that there is only a single broad tan $\delta$-peak in JD-400 (Fig. 5).

In all samples we observed also two additional peaks at lower temperatures, often denoted as $\beta$ - and $\gamma$-relaxations. They are related to motions of side groups and other local molecular motions and have been also observed in previous DMA 

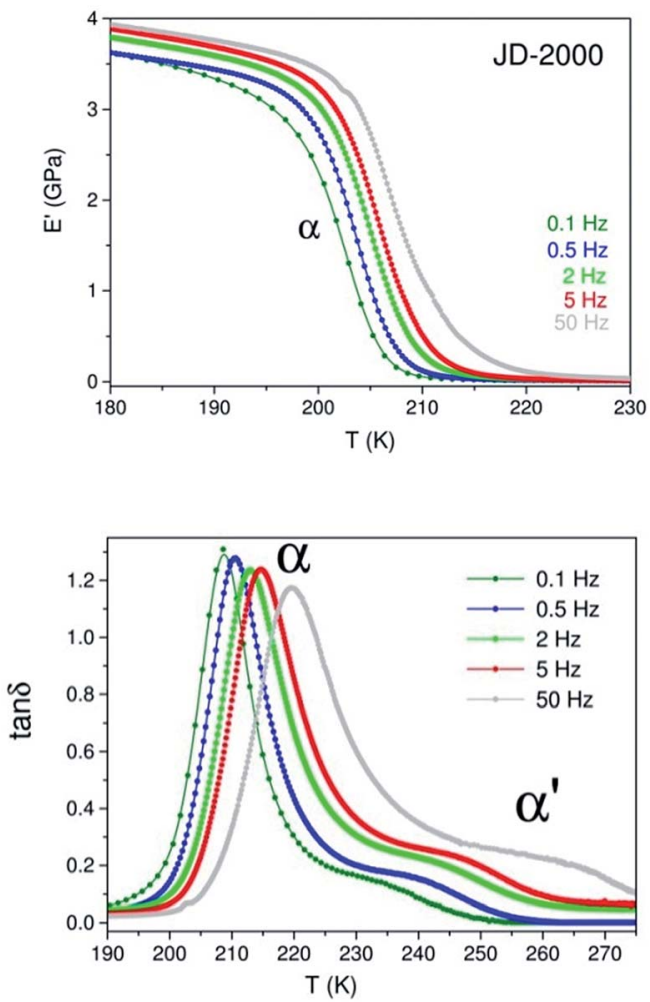

Fig. 3 Temperature dependence of storage modulus $E^{\prime}$ and $\tan \delta$ of polyurea JD-2000 at various frequencies.
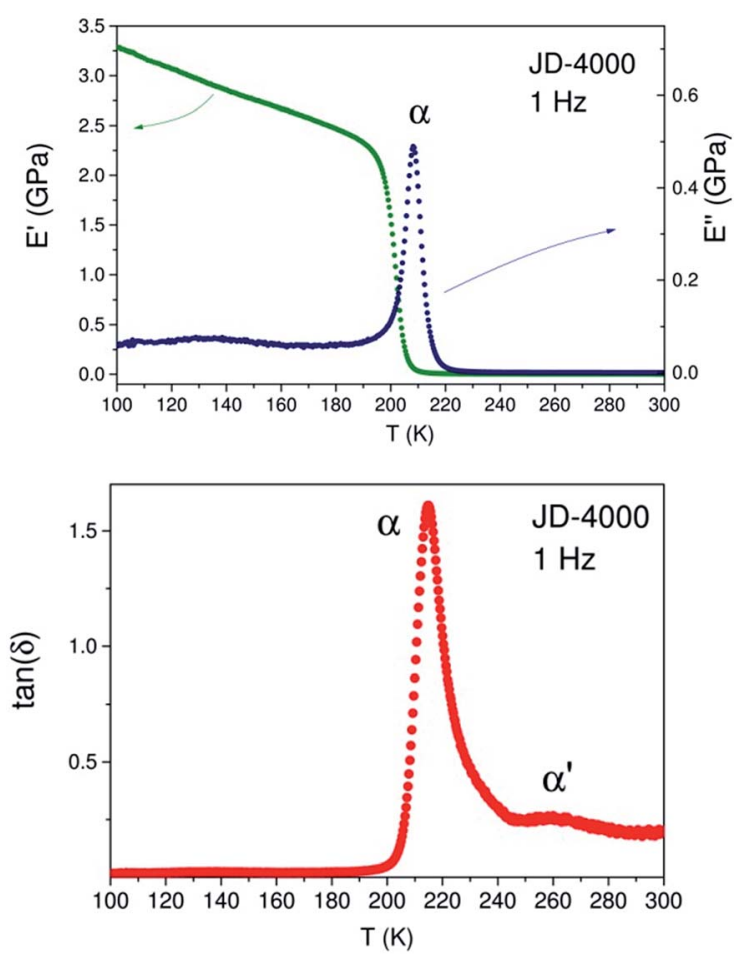

Fig. 4 Temperature dependence of storage modulus $E^{\prime}$, loss modulus $E^{\prime \prime}$ and $\tan \delta$ of polyurea JD-4000 measured at $f=1 \mathrm{~Hz}$.
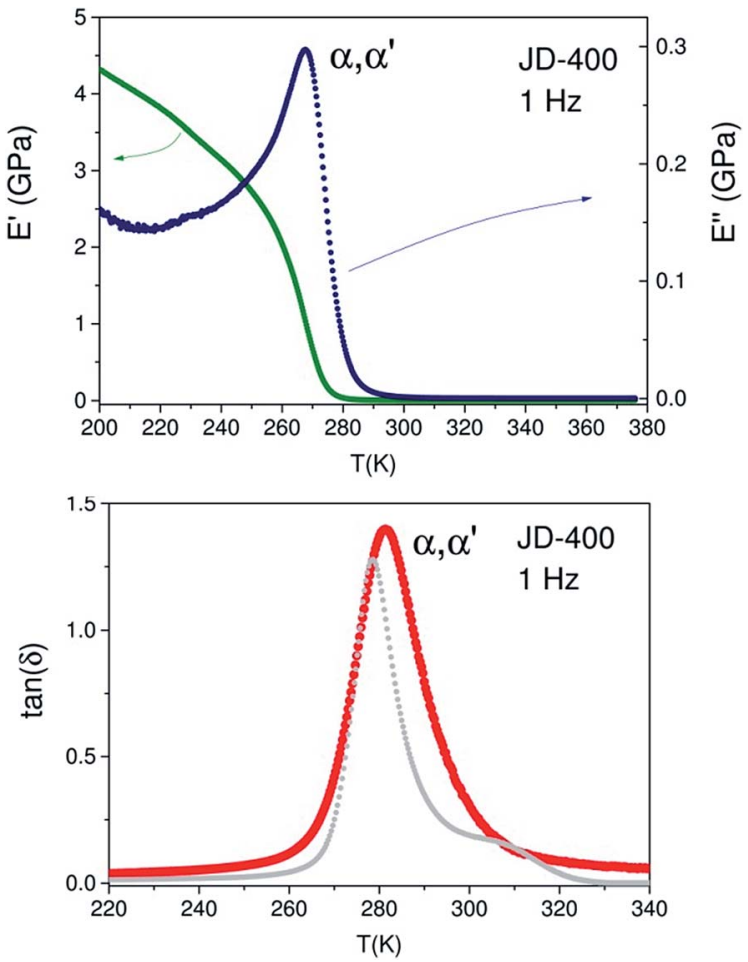

Fig. 5 Temperature dependence of storage modulus $E^{\prime}$, loss modulus $E^{\prime \prime}$ and $\tan \delta$ of polyurea JD-400 (red disks) measured at $f=1 \mathrm{~Hz}$. The grey disks display the shifted (with respect to 7 ) $\tan \delta$ of JD-2000 (at $0.5 \mathrm{~Hz}$ ) for comparison to emphasize the broadness of the JD-400 peak.

measurements of polyurea. ${ }^{32}$ As we focus on the $\alpha$ - and $\alpha^{\prime}$ process here we will show these details in a forthcoming paper.

\section{Discussion}

The segmental dynamics of polyurea has already been previously studied by dynamic mechanical analysis ${ }^{32,33}$ as well as by dielectric spectroscopy. ${ }^{34}$ Contrary to these $\mathrm{DMA}^{32,33}$ and specific heat ${ }^{34}$ measurements our DMA data clearly show (in tan $\delta$ of JD2000 and JD-4000) a second relaxation process $\left(\alpha^{\prime}\right)$ at a temperature higher than the $\alpha$-peak (Fig. 3 and 4). As we shall show below, for JD-400 (Fig. 5), the two glass transitions can only be separated by a careful fitting procedure.

In dielectric spectroscopy measurements ${ }^{34}$ a similar second relaxation process was found (denoted there also as $\alpha^{\prime}$-process) and turned out to be about 3 orders of magnitude slower than the $\alpha$-relaxation. Calorimetric data show ${ }^{34}$ that the $\alpha$-transition in polyurea is very broad with $\Delta T \approx 30-35 \mathrm{~K}$. This indicates a rather heterogeneous dynamics. Such a broad distribution of relaxation times can be accounted for by fitting a double Havriliak-Negami ${ }^{35}$ term for the $\alpha$ - and $\alpha^{\prime}$-relaxation to the data

$$
E^{*}(\omega)=E_{\infty}-\sum_{j=1}^{2} \frac{\Delta E_{j}}{\left[1+\left(i \omega \tau_{j}\right)^{\alpha_{j}}\right]^{\beta_{j}}}
$$

where $j=1,2$ correspond to the $\alpha$ - and $\alpha^{\prime}$-relaxation, respectively. $E_{\infty}$ is unrelaxed (high frequency limit) Young's modulus, $\Delta E_{1}=E_{\infty}-E_{\mathrm{s} 1}$ and $\Delta E_{2}=E_{\mathrm{s} 1}-E_{\mathrm{s} 2}\left(E_{\mathrm{s} 1}\right.$ and $E_{\mathrm{s} 2}$ are the static 
moduli of the corresponding process). The exponents $\alpha_{j}$ and $\beta_{j}$ describe the broadness and asymmetry of the corresponding spectra.

The temperature dependencies of the two relaxation times $\tau_{j}$ can be written in terms of the Vogel-Fulcher relationship as ${ }^{\mathbf{3 6}}$

$$
\tau_{j}=\tau_{0 j} \exp \left(\frac{B_{j}}{T-T_{0 j}}\right)
$$

where $\tau_{0 j}$ describe attempt rates, $B_{j}$ the activation energies and $T_{0 j}$ the corresponding Vogel-Fulcher temperatures of the $\alpha$-and $\alpha^{\prime}$-process.

Fig. 6, 7 and 8 show fits of $\tan \delta$ to the corresponding data of JD-2000, JD-4000 and JD-400 using eqn (3) and (4). The resulting fitting parameters are displayed in Table 2 .

Contrary to JD-2000 and JD-4000 the $\tan \delta$ curve of JD-400 exhibits only a single peak (Fig. 8), and hence does not obviously display two relaxation processes. However, it is impossible to fit the data assuming a single relaxation process in eqn (3). On the

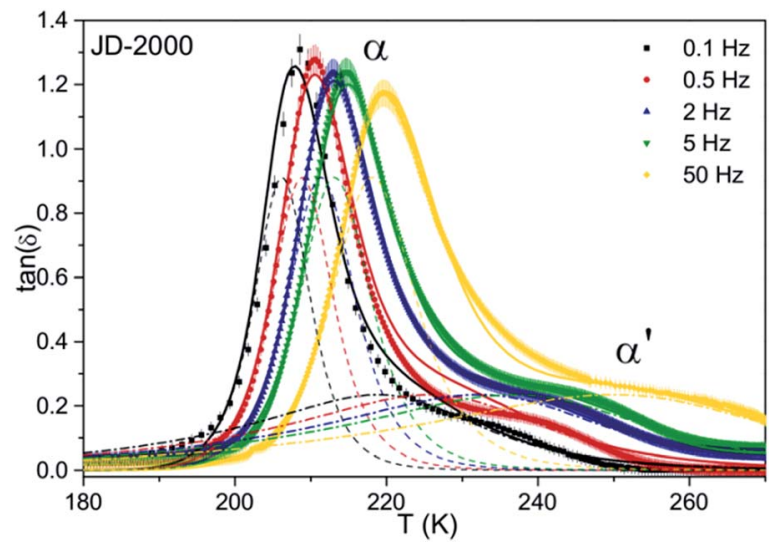

Fig. 6 Measured temperature dependence of $\tan \delta$ of polyurea JD2000 (disks). The full lines show a simultaneous fit for all measured frequencies with eqn (3) and (4). Dotted lines are generated by considering either the $\alpha$ - or the $\alpha^{\prime}$-relaxation.

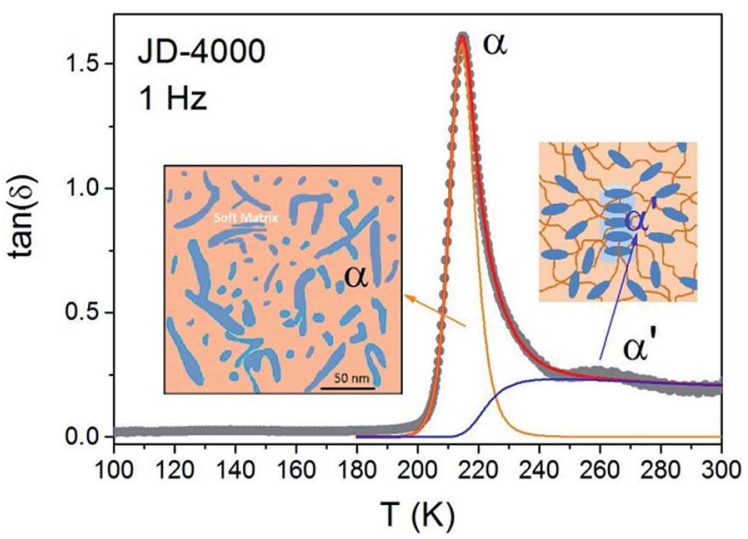

Fig. 7 Measured temperature dependence of $\tan \delta$ of polyurea JD4000 (grey disks). The red full line shows a fit with eqn (3) and (4). Orange and blue lines are generated by considering either the $\alpha$ - or $\alpha^{\prime}$ relaxation. The inset sketches the spatial regions of $\alpha$ and $\alpha^{\prime}$ relaxations.

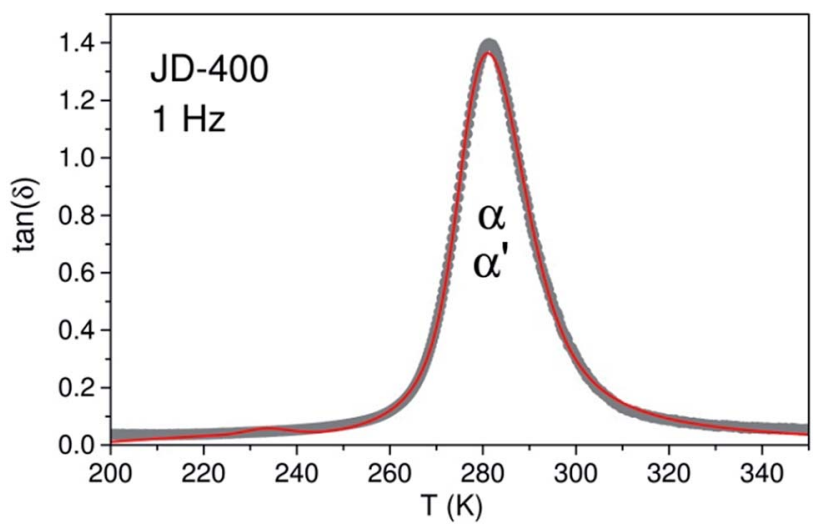

Fig. 8 Measured temperature dependence of $\tan \delta$ of polyurea JD400 (grey disks). The red line shows a fit with eqn (3) and (4). Here we do not show separate curves, since we cannot uniquely disentangle the $\alpha$ and $\alpha^{\prime}$-contributions.

other hand we cannot uniquely separate $\alpha$ and $\alpha^{\prime}$-contributions. Therefore we varied the relaxation contributions $\Delta E_{1}$ and $\Delta E_{2}$ in eqn (3), resulting in fits of similar quality characterized by chisquare values ranging from 0.28 to 0.39 . However, while $T_{\mathrm{g} 1}$ is determined with high accuracy the uncertainty of $T_{\mathrm{g} 2}$ is $\pm 10.6 \mathrm{~K}$.

Taking into account that mechanically measured relaxation times are usually shorter than dielectrically determined ones ${ }^{37}$ the fitting parameters in Table 2 are in reasonable agreement with those determined from dielectric spectroscopy data. ${ }^{34}$ Moreover, the main fitting parameters for the polyureas with different hard/soft segment ratios turn out to be rather similar.

In the following we will calculate the temperature dependence of dynamically correlated regions when approaching the glass transition in polyurea JD-2000 using the multipoint susceptibility approach of ref. 7 . In their work the authors proposed a very general but straightforward method to calculate a number of dynamically correlated units - which they call $N_{\text {corr, } 4}(T)$ - in glass forming materials. Relating un-measurable four-point correlations to an easily accessible response function, e.g. the temperature derivative of a dynamic two-point correlation function they obtain $^{38}$

Table $2 \alpha$ and $\alpha^{\prime}$ relaxation parameters for the three polyureas JD4000, JD-2000 and JD-400 obtained from fits with eqn (3) and (4)

\begin{tabular}{llll}
\hline & JD-4000 & JD-2000 & JD-400 \\
\hline$T_{01}(\mathrm{~K})$ & 156.6 & 153.8 & 221.5 \\
$\alpha 1$ & 0.78 & 0.7 & 0.66 \\
$\beta 1$ & 0.48 & 0.39 & 0.001 \\
$\tau_{01}(\mathrm{~s})$ & $10^{-15}$ & $10^{-15}$ & $10^{-15}$ \\
$B_{1}(\mathrm{~K})$ & 1755 & 1755 & 1755.2 \\
$\Delta E_{1} / E_{\infty}$ & 0.99 & 0.97 & 0.7 \\
$T_{02}(\mathrm{~K})$ & 206.3 & 92 & 171.8 \\
$\alpha 2$ & 0.16 & 0.57 & 0.61 \\
$\beta 2$ & 0.36 & 0.098 & 0.17 \\
$\tau_{02}(\mathrm{~s})$ & $10^{-13}$ & $10^{-13}$ & $10^{-13}$ \\
$B_{2}(\mathrm{~K})$ & 565.7 & 4010 & 1388 \\
$\Delta E_{2} / E_{\infty}$ & 0.0099 & 0.026 & 0.299
\end{tabular}




$$
N_{\text {corr }, 4}(T)=\frac{k_{\mathrm{B}} N_{\mathrm{A}}}{\Delta C_{\mathrm{P}} \bar{M}_{\mathrm{c}}} T^{2}\left\{\max _{\omega} \chi_{\mathrm{T}}(\omega, T)\right\}^{2}
$$

where $\Delta C_{\mathrm{P}}\left[\mathrm{J} \mathrm{g}^{-1} \mathrm{~K}^{-1}\right]$ is the specific heat anomaly at the glass transition temperature, $N_{\mathrm{A}}$ is the Avogadro constant, $\bar{M}_{\mathrm{c}}$ is the segmental molecular weight of the polyetheramines (soft segments) and $\chi_{\mathrm{T}}(\omega, T)=\left(1 / E_{\infty}\right) \mathrm{d} E^{\prime}(\omega, T) / \mathrm{d} T$ is the temperature derivative of a suitable correlation function (here the real part of the complex Young's modulus $E^{\prime}$ normalized with respect to $\left.E_{\infty}\right)$. In these units we obtain $N_{\text {corr, } 4}$ in terms of the number of correlated soft segments. To calculate an appropriate length scale $\xi$ we then have to multiply $N_{\text {corr, } 4}$ by the corresponding segmental length $l_{\mathrm{c}}$ (Table 1 ).

Fig. 9 shows the temperature derivatives of Young's modulus of JD-2000 as a function of frequency for various temperatures near the $\alpha$-glass transition. From the corresponding maxima, we have calculated $N_{\text {corr, } 4}(T)$, which is increasing with the decreasing temperature. Values of $N_{\text {corr, } 4}$ for many glass forming materials are reported in the literature, ${ }^{2,7,38}$ and it was shown that this quantity indeed increases when approaching $T_{\mathrm{g}}$, implying that dynamical correlations increase when approaching the glass transition.

It is nontrivial to relate the value of $N_{\text {corr }, 4}$ to certain values of spatially correlated regions. This is due to the fact that there is no need for the associated correlation volume $V_{\text {corr }}$ to be compact and therefore to scale as $V_{\text {corr }}=N_{\text {corr }, 4} / \rho \sim \xi^{3}$ as is the case for isotropic correlations. Although some theories, like e.g. the random first-order transition theory (RFOT) of glasses, predict that these correlated regions are compact, computer simulations suggest that CRRs are string-like..$^{\mathbf{8 , 9}, 39}$

To get a reliable estimation of the spatial correlations in polyurea we proceed as follows. Exploiting the approach described above, we calculate $N_{\text {corr }, 4}(T)\left(\right.$ with $^{34} \Delta C_{\mathrm{P}}=0.5 \mathrm{~J} \mathrm{~K}^{-1}$ $\mathrm{g}^{-1}$ ) and further the correlation volume $V_{\text {corr }}(T)$ (Fig. 10). At the laboratory glass transition $T_{\mathrm{g} 1}=198.6 \mathrm{~K}$, defined as $\tau_{1}\left(T_{\mathrm{g} 1}\right)=$ $100 \mathrm{~s}$, we obtain $V_{\text {corr }}\left(T_{\mathrm{g} 1}\right) \approx 10.2 \mathrm{~nm}^{3}$. Assuming a compact correlation volume and isotropic correlations this would imply $\xi$ $\approx V_{\text {corr }}^{1 / 3}$, leading to $\xi\left(T_{\mathrm{g} 1}\right) \approx 2 \mathrm{~nm}$. If however, we assume the

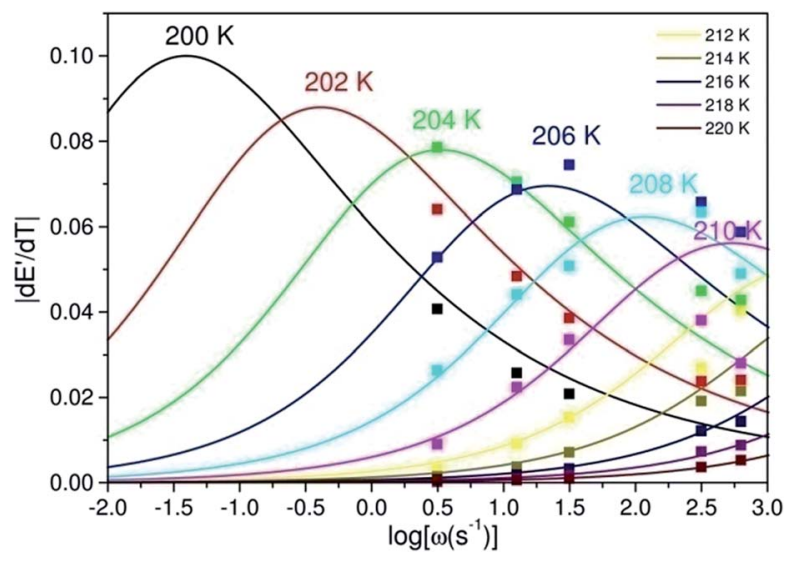

Fig. 9 Temperature derivatives of normalized Young's moduli as a function of frequency for various temperatures. Points are from experimental data of JD-2000 (Fig. 3) and lines are calculated from the corresponding fits to $E^{\prime}(\omega, T)$. dynamic correlations in polyurea to be string-like we can estimate their approximate length scale following two different approaches, which turn out to yield almost identical results (Fig. 10). For the first one, we assume that cylindrically shaped strings have an approximate thickness of a polyurea molecule, ${ }^{\mathbf{4 0}}$ i.e. $2 r \approx 0.56 \mathrm{~nm}$ - with $\xi=V_{\text {corr }} /\left(r^{2} \pi\right)$ - this leads to the blue disks in Fig. 10. For the second approach we calculate $\xi(T)$ (green disks in Fig. 10) from the number of correlated segments $N_{\text {corr,4 }}$ using eqn (5) with the corresponding segmental length $\left(l_{\mathrm{c}}=12.1 \mathrm{~nm}\right.$ for JD-2000) of Table 1 .

Fig. 10 shows the temperature dependencies of $V_{\text {corr }}$ and $\xi$. The points represent the experimentally determined values using eqn (5) and the procedures described above. The lines are fits using the relationship ${ }^{2,38}$

$$
V_{\text {corr }}(T)=\frac{N_{\text {corr }, 4}(T)}{\rho} \propto \frac{k_{\mathrm{B}}}{\rho \Delta C_{\mathrm{P}}} T^{2}\left(\frac{\mathrm{d} \ln \tau}{\mathrm{d} T}\right)^{2}
$$

which by using Vogel-Fulcher temperature dependence eqn (4) for $\tau(T)$ eqn (6) can be written as

$$
V_{\text {corr }}(T)=\frac{N_{\text {corr }, 4}(T)}{\rho} \propto \frac{k_{\mathrm{B}}}{\rho \Delta C_{\mathrm{P}}} T^{2} \frac{B^{2}}{\left(T-T_{0}\right)^{4}}
$$

leading to $\xi\left(T_{\mathrm{g} 1}\right) \approx 43 \mathrm{~nm}$.

After these data evaluations we arrive at the following picture for the two-glass transition behaviour of polyurea. For the $\alpha$ process dynamical correlations between molecules are increasing with decreasing temperature (Fig. 10) leading to a collective slowing down of (most probably string-like) molecular motions in the soft matrix.

We argue that the $\alpha^{\prime}$-process is related to a freezing of the motion of soft segments of restricted mobility in the vicinity of the hard domains for the following reasons: contrary to the $\alpha$ transition, the $\alpha^{\prime}$-transition affects the real part of the complex

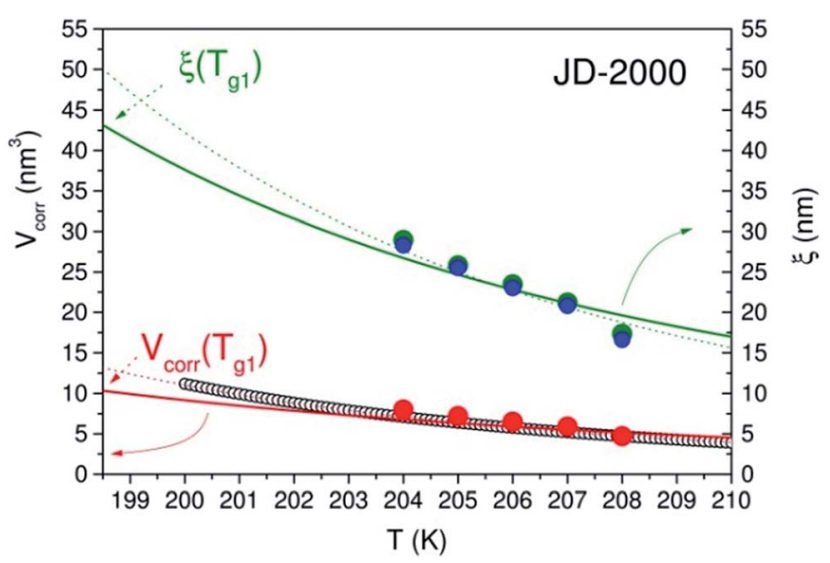

Fig. 10 Temperature dependencies of dynamically correlated regions approaching the $\alpha$-glass transition in polyurea JD-2000. The few full circles between 204 and $208 \mathrm{~K}$ are determined from the experimental values of $E^{\prime}(\omega, T)$ using eqn (5) together with $V_{\text {corr }}=N_{\text {corr }, 4} / \rho$ (red disks), $\xi=V_{\text {corr }} /\left(r^{2} \pi\right)$ (blue) and $\xi=l_{c} N_{\text {corr } 4}$ (green disks). The black open circles of $V_{\text {corr }}$ are determined by extrapolating the experimental data of $\mathrm{d} E^{\prime} / \mathrm{d} T$ by the fitted ones to $200 \mathrm{~K}$. The full lines are fits with eqn (7) using the fitting parameters $B_{1}$ and $T_{01}$ of Table 2. Dotted lines were obtained by varying the exponent in eqn (7) from 4 to 5.3 . 
Young's modulus only very little (from Table 2 we obtain $\Delta E_{2} \approx$ $0.027 \Delta E_{1}$ ), which is in agreement with the assumption that the $\alpha^{\prime}$-transition takes place in a very small shell surrounding the hard domains (Fig. 7).

The size of the hard nanodomains is of the order of $\approx 3-4$ $\mathrm{nm}$. This nanodomain size was calculated taking into account the volume fraction of both hard and soft domains, the average distance between two hard nanodomains (Table 1) which was obtained from the maximum intensity peak in the SAXS region $(d=2 \pi / q),{ }^{27}$ and the packing of spheres as an ideal model primitive cubic (PC), body-centered cubic (BCC) and facecentered cubic (FCC). The estimated radius of these hard domains is proportional to the product of the lattice parameter times the square root of the volume fraction of the crosslinker $\left(r \propto a \phi_{\mathrm{HD}}{ }^{1 / 2}\right)$; PC: $r=a\left[3 \phi_{\mathrm{HD}} /(4 \pi)\right]^{1 / 2}$, BCC: $r=a\left[3 \phi_{\mathrm{HD}} /(8 \pi)\right]^{1 / 2}$, and FCC: $r=a\left[3 \phi_{\mathrm{HD}} /(16 \pi)\right]^{1 / 2}$. From the calculated areas of $\tan \delta$ peaks with respect to the $\alpha$ - and $\alpha^{\prime}$-transition, we have estimated the thickness of the surrounding layer of restricted mobility to be of the order of $\lambda \approx 0.2 \mathrm{~nm}$ for JD-2000 when considering a packing of such model spheres with a hard-tohard domain distance of $d=5.4 \mathrm{~nm}$ and volume fractions of 0.04 and 0.84 for the polymer segment directly attached to the hard nanodomain interface and the bulk polymer, respectively.

The corresponding volume of this surrounding shell, which then is of the order of $\approx 30 \mathrm{~nm}^{3}$, is larger than the correlation volume $V_{\text {corr }}\left(T_{\mathrm{g} 1}\right) \approx 10 \mathrm{~nm}^{3}$ (Fig. 10). It implies that the region of restricted mobility is large enough to exhibit its own glass transition at $T_{\mathrm{g} 2}$. Similar estimations hold also for JD-4000 and for JD-400.

Using the fitting parameters (Table 2) one obtains (Fig. 11) that in the vicinity of the $\alpha^{\prime}$-peak the dynamics of these motions is indeed several orders of magnitude slower as compared to the dynamics of the polymer chain motions in the soft matrix $(\alpha-$ process).

Computer simulations ${ }^{15,23,41}$ show that the relaxations of chain segments in the vicinity of nanoparticles are necessarily slowed down, if there is an attractive interaction between nanoparticles and polymer chains. This is certainly

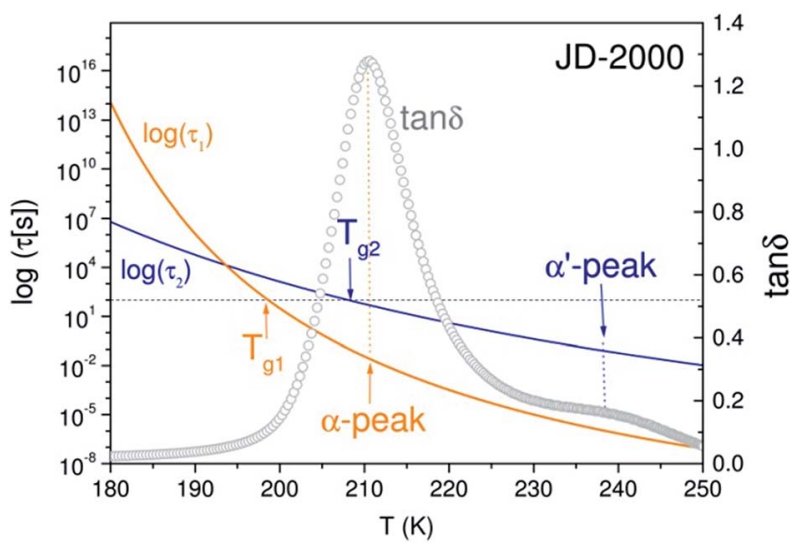

Fig. 11 Temperature dependencies of relaxation times $\tau_{1}$ and $\tau_{2}$ for JD-2000 calculated from eqn (4) with the parameters of Table 2. Tan $\delta$ was measured at $0.5 \mathrm{~Hz}$. the case here, since the soft segments (SS) are chemically attached to the hard domains. Under such conditions of permanent attachment PNCs have shown to exhibit two glass transition temperatures: ${ }^{42}$ one associated with polymer chains in the soft matrix far from the nanoparticles (hard domains in our case), and the other one at higher temperature is associated with chains in the vicinity of the nanoparticles (hard domains).

Table 3 gives the glass transition temperatures for the three polyureas obtained from the fitting parameters of Table 2 .

Fig. 12 shows the dependencies of the glass transition temperatures on the volume fractions $\phi_{\mathrm{x}}$ of the crosslinker (hard domains) and a fit with the relationship ${ }^{43}$

$$
T_{\mathrm{g} 1}=T_{\mathrm{g} 1}^{0}+k \frac{\phi_{\mathrm{x}}}{1-\phi_{\mathrm{x}}}
$$

Inspecting Fig. 12 we observe that around the critical volume fraction of $\phi_{\mathrm{x}}^{\mathrm{c}} \approx 0.19$ the two glass transitions were of reverse order, implying $T_{\mathrm{g} 1}=T_{\mathrm{g} 2}$ for $\phi_{\mathrm{x}}^{\mathrm{c}}$. This value of 0.19 corresponds to the percolation threshold of randomly oriented overlapping ellipsoids ${ }^{\mathbf{4 4}}$ of revolution with an aspect ratio of about $1: 4-$ $1: 5$. It implies that for $\phi_{\mathrm{x}}$ less than $19 \%$ the system consists of hard nanodomains embedded in a soft matrix. At $\phi_{\mathrm{x}}^{\mathrm{c}}$ the hard nanodomains percolate. For $\phi_{\mathrm{x}}>\phi_{\mathrm{x}}^{\mathrm{c}}$ the system consists of soft islands surrounded by a percolated network of hard nanodomains.

Table 3 Glass transition temperatures calculated from fitting parameters of Table 2 using $\tau\left(T_{\mathrm{g}}\right)=100 \mathrm{~s}$

\begin{tabular}{llc}
\hline Sample & $T_{\mathrm{g} 1}(\mathrm{~K})$ & $T_{\mathrm{g} 2}(\mathrm{~K})$ \\
\hline JD-400 & $266.3 \pm 0.2$ & $217.2 \pm 10.6$ \\
JD-2000 & $198.6 \pm 0.4$ & $208.2 \pm 2.1$ \\
JD-4000 & $201.4 \pm 0.2$ & $222 \pm 1.4$
\end{tabular}

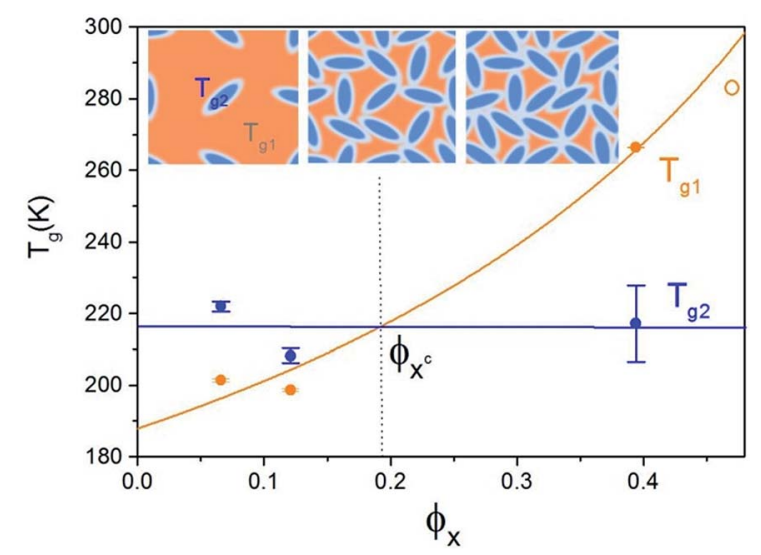

Fig. 12 Glass transition temperatures of three polyurea networks JD4000, JD-2000 and JD-400 vs. volume fractions $\phi_{x}$ of the hard nanodomains. The orange line is a fit with eqn (8), yielding $T_{\mathrm{g} 1}^{0}=(187.9$ $\pm 6.6) \mathrm{K}$ and $k=(119.6 \pm 17.2) \mathrm{K}$. The polyurea elastomer ${ }^{27} \mathrm{ET}-403$ with a volume fraction of $\phi_{\mathrm{x}}=0.47$ (orange open circle) follows the trend rather well. 


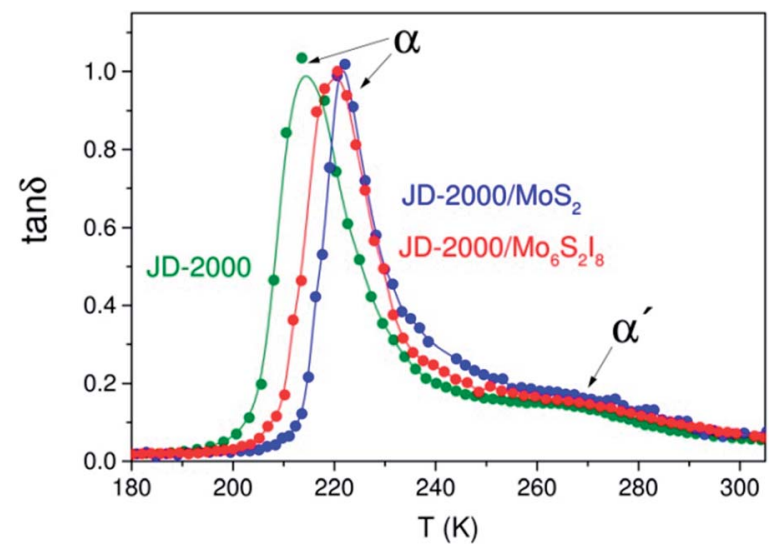

Fig. 13 Tan $\delta$ of pure polyurea JD-2000 compared with the nanocomposites JD-2000/MoS 2 and JD-2000/Mo $\mathrm{S}_{2} \mathrm{I}_{8}$ (1 wt\% of $\mathrm{MoS}_{2}$ and $\mathrm{Mo}_{6} \mathrm{~S}_{2} \mathrm{I}_{8}$ ). The measurements were performed at a frequency of $1 \mathrm{~Hz}$.

Finally we will discuss the physical origin of the strong increase of $T_{\mathrm{g} 1}$ with increasing $\phi_{\mathrm{x}}$. Because the size $\xi$ of CRRs increases for $T \rightarrow T_{\mathrm{g} 1}$ (Fig. 10), the molecules of polymer chains at distances $r \leq \xi$ away from the surface of the hard nanodomains will "feel" the surface induced slowing down. Since the average distance between the hard nanodomains is smaller than $\xi\left(T_{\mathrm{g} 1}\right) \approx 43 \mathrm{~nm}$, the dynamics of the soft domains is considerably slowed down, rationalizing the observed increase of the glass transition temperature.

A very similar behaviour was recently found for nanocomposites of polyurea JD-2000 filled with $1 \mathrm{wt} \%$ of $\mathrm{MoS}_{2}$ nanotubes and $\mathrm{Mo}_{6} \mathrm{~S}_{2} \mathrm{I}_{8}$ nanowires. ${ }^{45}$ Although the inorganic nanoparticles are much larger (100 nm diameter, $10 \mu \mathrm{m}$ length) and their concentration is 1-2 orders of magnitude smaller as compared to the hard nanodomains, a clear increase of $T_{\mathrm{g} 1}$ was observed in the presence of nanoparticles, whereas $T_{\mathrm{g} 2}$ remained constant (Fig. 13). Also in this case the increase of $T_{\mathrm{g} 1}$ results from a slowing down of molecular motions near the nanoparticle surface, which due to an increase of the correlation length $\xi$ extends deeply into the matrix.

\section{Summary and conclusions}

We have studied the glass transitions in microphase segregated polyurea networks by measuring the dynamic elastic susceptibility in a broad temperature range and at frequencies between 0.1 and $100 \mathrm{~Hz}$. By varying the chain length $l_{\mathrm{c}}$ of the soft part of the polyurea molecules between $24.5 \mathrm{~nm}$ and $2.5 \mathrm{~nm}$, the volume fractions $\phi_{\mathrm{x}}$ of hard nanodomains increased from $7 \%$ to $39 \%$, implying that the average distance $t$ between the hard nanodomains decreases with decreasing chain length $l_{\mathrm{c}}$.

Two distinct peaks in $\tan \delta$ were clearly resolved at $T_{\alpha}$ and $T_{\alpha^{\prime}}$ $>T_{\alpha}$ for samples with 7 and 12 vol\% of hard nanodomains. They were attributed to glass transitions in the soft matrix ( $\alpha$-peak) and at the interface between the hard nanodomains and the matrix ( $\alpha^{\prime}$-peak), respectively. We have fitted the two tan $\delta$ peaks with two Havriliak-Negami terms and Vogel-Fulcher dependencies of the corresponding relaxation times $\tau_{\alpha}$ and $\tau_{\alpha^{\prime}}$. Using these fitting parameters we have determined the glass transition temperatures $T_{\mathrm{g} 1}\left(\phi_{\mathrm{x}}\right)$ and $T_{\mathrm{g} 2}\left(\phi_{\mathrm{x}}\right)$, corresponding to the $\alpha$ and $\alpha^{\prime}$-process, respectively. It turns out that $T_{\mathrm{g} 1}$ increases strongly with increasing volume fraction of the hard nanodomains, whereas $T_{\mathrm{g} 2}$ remains almost constant.

The strong increase of $T_{\mathrm{g} 1}$ with increasing $\phi_{\mathrm{x}}$ results from the fact that due to the observed strong increase of a dynamical correlation length $\xi$ with $T \rightarrow T_{\mathrm{g} 1}$, the interfacially slowed down regions near the hard nanodomains extend deep into the soft matrix, and the longest relaxation time $\tau_{\alpha}$ increases, resulting in an increase of the average $T_{\mathrm{g} 1}$. The effect increases with increasing density of the nanodomains. Molecular dynamics simulations ${ }^{15,23,39,46}$ corroborate such a picture. On the other hand the molecules near the hard nanodomains are already strongly (chemically) attached, so that their dynamics is substantially slowed down and thus $T_{\mathrm{g}_{2}}$ is not expected to be much influenced by an increase of $\phi_{\mathrm{x}}$, in agreement with the observations.

At the critical value of $\phi_{\mathrm{x}}^{\mathrm{c}} \approx 0.19$ the two glass transition temperature curves intersect, implying that at this concentration of hard nanodomains one cannot longer separate the dynamics of molecular motions close to the nanodomain interface from that of the soft matrix. At this critical concentration, the hard nanodomains start percolating and microphase segregated polyurea changes from a system of hard nanodomains embedded in a soft matrix to the opposite case of a soft polymer confined in a network of interconnected hard domains. This is also accompanied by a strong reinforcement effect, i.e. Young's modulus at $100 \mathrm{~K}$ changes from $4 \mathrm{GPa}$ (for $\phi_{\mathrm{x}}$ $=0.07$ ) to $8 \mathrm{GPa}$ (for $\phi_{\mathrm{x}}=0.39$ ). The critical concentration of about 0.19 corresponds to the percolation threshold ${ }^{44}$ of overlapping ellipsoids with an aspect ratio between $1: 4$ and $1: 5$. Of course such a simple geometrical model has at this stage to be taken with caution. E.g. if instead of rotational ellipsoids one would take overlapping spherocylinders, the observed percolation threshold ${ }^{47}$ would imply $D / L \approx 1: 2-1: 3$ ( $D=$ diameter, $L$ $=$ length of the spherocylinder). Although such shapes of nanodomains are in reasonable agreement with some previous AFM measurements, ${ }^{28}$ more detailed investigations are needed to test these predictions which are based on a rather idealized geometrical model.

Summarizing, our findings lead to interesting insights into the two-glass transition behaviour of microphase segregated polyurea which are valid for other NPCs, too. However, as they are based on a rather macroscopic approach and phenomenological analysis they should be complemented by microscopic measurements as well as computer simulations. Detailed AFM measurements are required to reveal the morphologies of nanodomains in polyureas with different hard/soft segment ratios of the chains and to determine the corresponding average distances between the hard nanodomains as well as their exact shape. Molecular dynamics simulations of the type of ref. 39 could help to considerably improve the quality of fitting of dynamic susceptibility data. At present we know that in the vicinity of nanoparticles $\tau(r, T)=\tau_{0}(r) \exp \{\xi(r, T) \Delta(r) / T\}$ with $\tau(r \rightarrow \infty)>\tau$ (pure polymer) and we do not have a clue on the specific $r$-dependencies of $\tau_{0}, B$ and $T_{0}$ in the corresponding 
Vogel-Fulcher equation. Knowledge of the spatial dependence of the relaxation time in the vicinity of a nanoparticle - like e.g. it is shown in Fig. 11 of ref. 39 - would help to reduce the number of · fitting parameters considerably.

In addition to the obtained fundamental insights, the present work yields results that may be also interesting from the application point of view: the properties of polyurea can be easily tuned by varying the segmental molecular weight of polyetheramines, i.e. the volume fraction $\phi_{\mathrm{x}}$ of hard nanodomains. The glass transition temperature increases from $-75^{\circ} \mathrm{C}$ for $\phi_{\mathrm{x}}=$ 0.07 to $-7{ }^{\circ} \mathrm{C}$ for $\phi_{\mathrm{x}}=0.39$, i.e. with decreasing molecular weight and is accompanied by a strong reinforcement effect of $100 \%$. In principle these properties could be further improved if the density of nanodomains could be still increased. It has been shown that packing fractions of more than 0.7 can be reached for ellipsoids ${ }^{48}$ and superellipsoids. ${ }^{49}$ Inserting this value into eqn (8) one obtains a theoretical glass transition temperature of $194{ }^{\circ} \mathrm{C}$. Although such high values may not be reached in practice, the polyurea elastomer ET-403 (ref. 27) with a volume fraction of $\phi_{\mathrm{x}}=0.47$ yields a glass transition temperature of +10 ${ }^{\circ} \mathrm{C}$, which is well predicted by eqn (8) with the fitting parameters of the present work (Fig. 12).

\section{Acknowledgements}

The present work was performed in the frame of the COST Action MP0902 (COINAPO - Composites of Inorganic Nanotubes and Polymers). Financial support from the Austrian Science Fund (FWF) P23982-N20 is gratefully acknowledged.

\section{References}

1 G. Adam and J. H. Gibbs, J. Chem. Phys., 1965, 43, 139.

2 S. Capaccioli, G. Ruocco and F. Zamponi, J. Phys. Chem. B, 2008, 112, 10652.

3 P. Debenedetti and F. H. Stillinger, Nature, 2001, 410, 259.

4 G. Hempel, A. Hempel, A. Hensel, C. Schick and E. Donth, J. Phys. Chem. B, 2000, 104, 2460.

5 S. A. Reinsberg, A. Heuer, B. Doliwa, H. Zimmermann and H. W. Spiess, J. Non-Cryst. Solids, 2002, 307-310, 208.

6 X. H. Qiu and M. D. Edinger, J. Phys. Chem. B, 2003, 107, 459. 7 L. Berthier, G. Biroli, J.-P. Bouchard, L. Cipelletti, D. ElMasri, D. L'Hote, F. Ladieu and M. Pierno, Science, 2005, 310, 1797.

8 C. Donati, J. F. Douglas, W. Kob, S. J. Plimpton, P. H. Poole and S. C. Glotzer, Phys. Rev. Lett., 1998, 80, 2338.

9 J. D. Stevenson, J. Schmalian and P. G. Wolynes, Nat. Phys., 2006, 2, 268.

10 J. A. Forrest and J. Mattsson, Phys. Rev. E: Stat. Phys., Plasmas, Fluids, Relat. Interdiscip. Top., 2000, 61, R53.

11 B. Jérome and J. Commandeur, Nature, 1997, 386, 589.

12 P. Pissis, A. Kyritsis, D. Daoukaki, G. Barut, R. Pelster and G. Nimtz, J. Phys.: Condens. Matter, 1998, 10, 6205.

13 C. L. Jackson and G. B. McKenna, Chem. Mater., 1996, 8, 2128.

14 W. Schranz, M. R. Puica, J. Koppensteiner, H. Kabelka and A. V. Kityk, Europhys. Lett., 2007, 79, 36003.
15 P. Scheidler, W. Kob and K. Binder, Europhys. Lett., 2000, 52, 277.

16 P. Scheidler, W. Kob and K. Binder, Europhys. Lett., 2002, 59, 701.

17 O. Trofymluk, A. A. Levchenko and A. Navrotsky, J. Chem. Phys., 2005, 123, 194509.

18 J.-Y. Park and G. B. McKenna, Phys. Rev. B: Condens. Matter Mater. Phys., 2000, 61, 6667.

19 C. LeQuellec, G. Dosseh, F. Audonnet, N. Brodie-Lindner, C. Alba-Simionesco, W. Häussler and B. Frick, Eur. Phys. J.: Spec. Top., 2007, 141, 11.

20 M. Alcoutlabi and G. B. McKenna, J. Phys.: Condens. Matter, 2005, 17, R461.

21 C. Alba-Simionesco, B. Coasne, G. Dosseh, G. Dudziak, K. E. Gubbins, R. Radhakrishnan and M. SliwinskaBartkowiak, J. Phys.: Condens. Matter, 2006, 18, R15.

22 H. Oh and P. F. Green, Nat. Mater., 2009, 8, 139.

23 F. W. Starr, T. B. Schroder and S. Glotzer, Macromolecules, 2002, 35, 4481.

24 G. D. Smith, D. Bedrov, L. Li and O. Byutner, J. Chem. Phys., 2002, 117, 9478.

25 G. Tsagaropoulos and A. Eisenberg, Macromolecules, 1995, 28, 6067.

26 J. M. Kropka, V. Pryamitsyn and V. Ganesan, Phys. Rev. Lett., 2008, 101, 075702.

27 A. Sánchez-Ferrer, D. Rogez and P. Martinoty, Macromol. Chem. Phys., 2010, 211, 1712.

28 M. Grujicic, B. Pandurangan, T. He, B. A. Cheeseman, C.-F. Yen and C. L. Randow, Mater. Sci. Eng., A, 2010, 527, 7741.

29 J. Koppensteiner, W. Schranz and M. R. Puica, Phys. Rev. B: Condens. Matter Mater. Phys., 2008, 78, 054203.

30 J. Koppensteiner, W. Schranz and M. A. Carpenter, Phys. Rev. B: Condens. Matter Mater. Phys., 2010, 81, 024202.

31 W. Schranz, Phase Transitions, 1997, 64, 103.

32 J. Yi, M. C. Boyce, G. F. Lee and E. Balizer, Polymer, 2006, 47, 319.

33 J. A. Pathak, J. N. Twigg, K. E. Nugent, D. L. Ho, E. K. Lin, P. H. Mott, C. G. Robertson, M. K. Vukmir, T. H. Epps III and C. M. Roland, Macromolecules, 2008, 41, 7543.

34 D. Fragiadakis, R. Gamache, R. B. Bogoslovov and C. M. Roland, Polymer, 2010, 51, 178.

35 S. Havriliak and S. Negami, J. Polym. Sci., Part C: Polym. Symp., 1966, 14PC, 99.

36 F. Kremer and A. Schönhals, Broadband dielectric spectroscopy, Springer, Berlin, New York, 2003.

37 N. G. McCrum, B. E. Read and G. Williams, Anelastic and dielectric effects in polymeric solids, Dover Publications, New York, 1991.

38 C. Dalle-Ferrier, C. Thibierge, C. Alba-Simionesco, L. Berthier, G. Biroli, J. P. Bouchaud, F. Ladieu, D. L'Hote and G. Tarjus, Phys. Rev. E: Stat., Nonlinear, Soft Matter Phys., 2007, 76, 041510.

39 B. A. Pazmino Betancourt, J. F. Douglas and F. W. Starr, Soft Matter, 2013, 9, 241.

40 P. D. Godfrey, R. D. Brown and A. N. Hunter, J. Mol. Struct., 1997, 413-414, 405. 
41 M. Vacatello, Macromolecules, 2001, 34, 1946.

42 G. Tsagaropoulos and A. Eisenberg, Macromolecules, 1995, 28, 396.

43 H. Stutz, K.-H. Illers and J. Mertes, J. Polym. Sci., Part B: Polym. Phys., 1990, 28, 1483.

44 E. J. Garboczi, K. A. Snyder, J. F. Douglas and M. F. Thorpe, Phys. Rev. E: Stat. Phys., Plasmas, Fluids, Relat. Interdiscip. Top., 1995, 52, 819.
45 M. Reinecker, A. Fuith, V. Soprunyuk, A. Sánchez-Ferrer, A. Mrzel, R. Torre and W. Schranz, Phys. Status Solidi A, 2013, 210, 2320.

46 M. Vacatello, Macromolecules, 2001, 34, 1946.

47 B. Nigro, C. Grimaldi, P. Ryser, A. P. Chatterjee and P. van der Schoot, Phys. Rev. Lett., 2013, 110, 015701.

48 A. Donev, F. H. Stillinger, P. M. Chaikin and S. Torquato, Phys. Rev. Lett., 2004, 92, 255506.

49 G. W. Delaney and P. W. Cleary, Europhys. Lett., 2010, 89, 34002 . 